\title{
VISUALIZATION OF MEMS STRUCTURES FOR EDUCATION OF AUTOMOBILE MICROSYSTEMS
}

\author{
Slavomír KARDOŠ゙ \\ *Department of Technologies in Electronics, Faculty of Electrical Engineering and Informatics, \\ Technical University of Košice, Letná 9, 04200 Košice, Slovak Republic, Tel.: +421 55602 3211, E-mail: slavomir.kardos@tuke.sk \\ ${ }^{* *}$ Department of Electrotechnology, Faculty of Electrical Engineering, Czech Technical University in Prague, Technická 2, \\ 16627 Praha, Czech Republic, Tel.: +420 22435 2353, E-mail: busekd1@fel.cvut.cz
}

\begin{abstract}
The work is aimed at visualization of working principles of microelectromechanical sensors which are used in automobile electronic systems. Attention is paid to accelerometric, inclinometric, pressure and MAF devices. These are used in systems for stability control, passive and active safety as well as comfort management. The visualizations were realized using modern animation techniques initiated by the creation of virtual materials, $2 \mathrm{D}$ and $3 \mathrm{D}$ objects, through scene exposure, pending their animation and final cutting.
\end{abstract}

Keywords: MEMS, accelerometer, inclinometer, pressure sensor, HUD, MAF, LIGA, micromachining

\section{INTRODUCTION}

Application of modern technologies was preceded by continuous development which was stimulated by demands for high functionality, low price and high reliability. Development in automobile industry continually accelerates and especially the problematics of Microelectromechanical systems (MEMS) is considerably current. Innovations in the automobile systems are significantly based on application of MEMS technologies.

(MEMS) are micromechanical systems joined with electronic systems. They are produced by micromachining processes similar to the realization technologies of microelectronic devices. The micromachining is based on deposition and removal of layers. The processes can have microlayer scope as well as deep scope. The MEMS consists of mechanical parts, sensors, actuators and other components which are obviously integrated on single common substrate, mainly on silicon.

MEMS based sensors are efficient for measurement of thermal, chemical, mechanical, biological, optical, magnetic and other parameters. Integrated electronics is intended for transformation into electric signal. Incorporated electronics controls the actuators by adjustment of position, pumping, movement, regulation, filtration and other processing to attain desired functionalities.

The scale of MEMS components ranges from fraction of one nanometer to fraction of one millimeter. Main advantages of MEMS devices are therefore small dimensions, low weight, easy system integration possibility, high resistance against mechanical shocks, vibrations, radiation and an easy implementation into a mass production.

In automobile industry, the MEMS are used in airbag systems, systems for automobile security, for detection of driving parameters, navigation, engine control, they are used in headlights, for dynamic stability control, pressure measurement and in many other systems.

In consumer electronics, they are used in home equipment, sporting systems, computer components, navigation systems, active subwoofers, drones and so on.
Other industry sectors use the MEMS for detection of earthquakes, in natural gas valves, for quality evaluation of equipment. In military they are used in navigation and interception systems, accessories for soldiers. In biotechnologies, such systems are used in biochips, for the measurement of blood pressure, in Lab-on-Chip technologies, biosensors and chemical sensors.

Most used sensing principles in MEMS systems are resonant, capacitive (Fig. 1), piezoresistive, piezoelectric, thermoelectric and optical. [1]

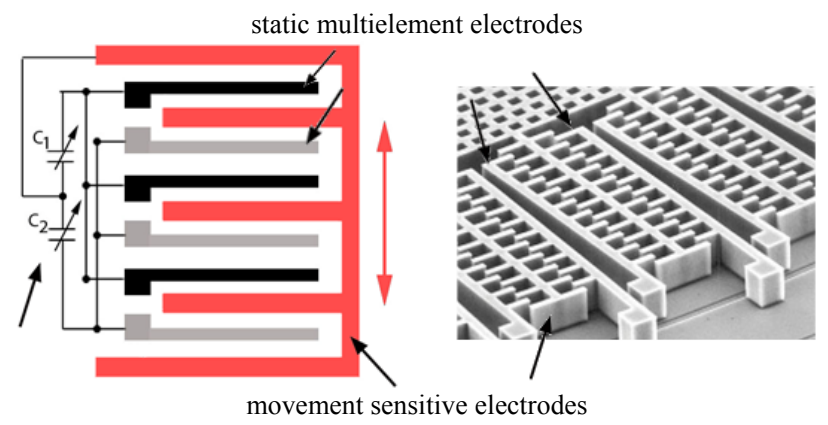

Fig. 1 Multielectrode capacitive sensor in bridge arrangement (left) [2], capacitive accelerometer microstructure (right) [3]

Widely used LIGA technology (Lithographie, Galvanoformung, Abformung, i.e. Lithography, Electroplating, and Molding) allows space scale forming of ceramics, metals, polymers and glasses for MEMS system elements. Another technology, surface micromachining allows forming of surface structures including complex microelectronic sensor structures. Volume micromachining is the technology used for ink-jet printer heads as well as components like membranes for pressure sensors. During MEMS structures forming, the excimer laser is frequently used for polymer materials treatment. [4]

\section{VISUALIZATION SOFTWARE}

Elementary models were created using Autodesk 3DS Max software. It is a modelling tool using which various 
methods are possible for objects modelling. In this work, all models were realized by "edit poly" method, i.e. with geometric multi-edge elements. The "key frames" method was used for the animation of rotation, scaling and movement of an object in space and time using key frame locking. These frames are simply joined by curves which regulate rotation and movement of objects along the timeline in the space. [7]

Scenes lighting, surfaces of materials and camera were realized by V-ray package tool. This tool is a rendering "ray tracing" engine as a plug-in of 3DS Max software. The surfaces of plastic and metallic materials were created using this software. It is possible to realize the photorealistic visualizations thanks to the simulation of real incidence and reflection of light photons including behavior of real camera objective. Various types of materials can be simulated using this software.

Editing and management of bitmap objects was realized using Adobe Photoshop CS6 software.

As a digital video cutting tool, the Adobe Premiére CS6 was used. The visualizations were composed of several variously created modules which were joined and properly timed.

The Adobe After Effects, a tool for realization of 2D animations and visual effects, was used for visualization of the components which were not efficient to be created by $3 \mathrm{D}$ approach. This tool was also used for "motion capture" which was needed for tracing of the movement of specified point in the moving scene.

\section{MODELLING OF OBJECTS AND VISUALIZATION PRACTICES}

Realized visualizations of MEMS devices working principles are mostly composed of three sections. In left window, the automobile with simulated situation is visualized. In upper right window, the detail of particular MEMS sensor is visualized with simulated influence of main physical parameters at the sensor structure. In lower right window, the graphical interpretation of sensed parameter is synchronous with the scene. This process was realized by synchronization of particular 3D renders from 3DS Max software and 2D renders from After Effects software for additional effects and graphs.

Modelling process was realized using tools of "edit poly" method in 3DS Max as was yet mentioned. The base of every model was initiated from a fundamental object (cube, sphere or cylinder type), which is treated by additional topology into grid structure of an object, followed by extending and trimming into new shapes. For fine rounded edges of the objects, the "turbosmooth" tool was utilized. This tool uses quadratic multiplication of an object grid structure and the curves averaging. The objects in "edit poly" consist of five basic editable components vertex, edge, border, polygon and element. The vertexes are peaks or points which join particular polygons in the $3 \mathrm{D}$ object. The edge joins the two vertexes. The border is established at the edge of open areas. Closed object does not contain any borders of course what is needed for $3 \mathrm{D}$ print data. The polygon is an area between the edges. The element is a self-contained object.

Objects topology editing was realized by main tools as Move, Rotate and Scale as well as additional tools as
Attach, Bridge, Collapse, Connect detach and Extrude (Fig. 2). The Move tool was used for moving of selected objects in space along $\mathrm{X}, \mathrm{Y}$ and $\mathrm{Z}$ axes. The Rotate tool was used for rotation of the components in space. The Scale allowed enlarging and shrinking selected structures. The Bridge allowed bridging between two selected polygons. The Collapse was used for merging of more vertexes into single one. The Connect allowed topological transection by selected edges of an object. The Extrude was used for extending and interjecting of selected polygon as well as bridged topology forming. The Attach allowed merging of objects into single one with original objects as elements. The Detach was used for elements expanding into object form. [7]
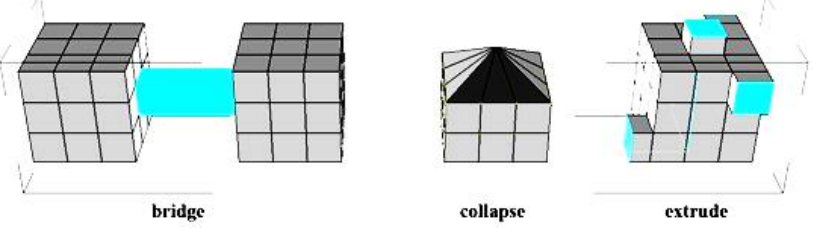

Fig. 2 The products of bridge, collapse a extrude process [7]

\subsection{Material surfaces}

The materials were composed using V-ray plug-in raytracing engine which is efficient to simulate the real materials as metals, plastics, glass, human skin, hair and many others. The V-ray includes the five main methods which influence the properties of a created material. These are the Diffuse, Self-illumination, Reflection, Refraction and Translucency. The Diffuse allowed using RGB palette to set the base material color. The Self-illumination allowed setting the color and intensity of light which is emitted from the material's surface. The Reflection allowed setting of the sleekness of the material, i.e. the ability to reflex the incident light. The Refraction allowed setting of the color and intensity of light transmitted through the material, its dispersion as well as its refraction index. Finally, the Translucency allowed setting of the method of light absorption.

\subsection{Lighting and camera}

In addition to materials composing, the V-ray plug-in allows to apply the lights including simulation of sunlight. It was utilized at visualization of the automobile in daylight as well as for studio lighting at MEMS structures detail view. In V-ray engine, the camera was simulated according to real camera parameters. Used simulated camera had $36 \mathrm{~mm}$ negative width (film gate), $40 \mathrm{~mm}$ focal distance (focal length), 1/200 s shutter speed, aperture (f-number) of 8 and ISO of 100. The white color correction was off at animations that used flat studio lights. The light blue was used for sunny climate simulation.

\subsection{D animations composing}

The animations of objects movement by 3DS Max software were created using key frames. The initial and final position of an object was selected together with initial and final movement time by locking of the key 
frames in specified states. Then the smooth transition between them was generated.

$3 \mathrm{D}$ animations rendering with simulation of real materials, light sources and cameras it very much time consuming process during which the V-ray engine calculate the light photons behavior at incidence, diffusion, reflexing, transmission through various materials and at incidence at simulated camera lens. For optimization of rendering time, some particular processes during animation as well as additional effects were realized using After Effects and Premiere software which rendering time only in $2 \mathrm{D}$ space is markedly lower.

\section{4. $2 \mathrm{D}$ animations composing}

The animations of graphs and mentioned additional effects to the $3 \mathrm{D}$ animations were realized by After Effects software. Initially, a multilayer picture structure was created by Photoshop CS6 software. At the time line, the animations were composed using key frames just as at 3D animations. By adjustment of overlaying, visibility, movement and rotation of particular layers as well as by synchronization of frames according to rendered 3D animations, the graphs were composed with content according to MEMS device behavior in a simulated automobile.

Rendering of 2D animations was the process during which the picture sequences were recorded according to the animated 2D objects (composed of picture layers).

\subsection{Digital cut of the video sequences}

Ever visualization consists of a set of modules which are synchronized and arranged. For that purpose the Adobe Premiére digital cut software was utilized. This software was also used for the final rendering of video sequences into the format which is appropriate for streamed HD video.

\section{PRACTICAL VISUALIZATIONS}

Composed video visualizations bring detailed view on particular MEMS sensors which are used in automobile electronic systems. They are intended to support the education process in the frame of study objects of MSc. study. The animated visualizations practically explain the MEMS structures and the physical principles of the particular system. They are supported by additional text and graphical information which is dynamically updated.

In Fig. 3, the MEMS inclinometer response in driveoff assistant system is depicted. The screenshot from visualization imagine the stalled automobile stimulated by the assistant with information about inclination. In Fig. 4, the MEMS accelerometer response of airbag system in idle state closely before and in time of an impact momentum with a barrier is depicted. Fig. 5 is a screenshot from the head-up display utilizing MEMS scanner. In Fig. 6, the measurement of mass-flow utilizing MEMS MAF sensor is depicted. Finally, in Fig. 7, the visualization of a measurement of tire pressure utilizing MEMS pressure gauge is presented by screenshots of two different pressure states.

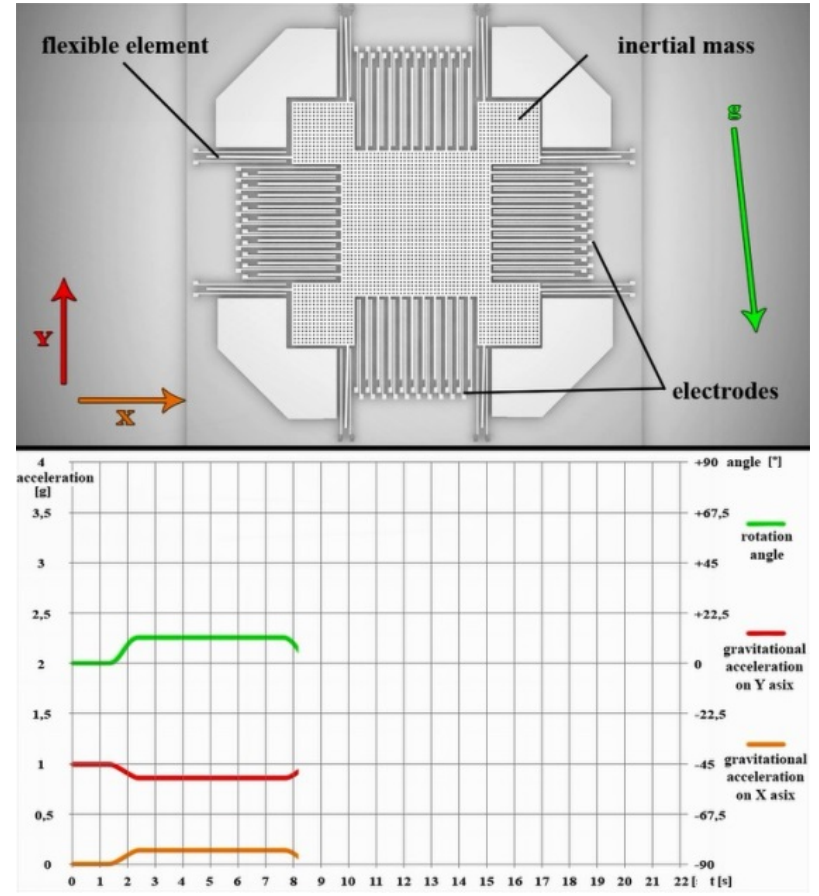

Fig. 3 MEMS inclinometer response applied in drive-off assistant system - stalled automobile stimulated by the assistant with information about inclination

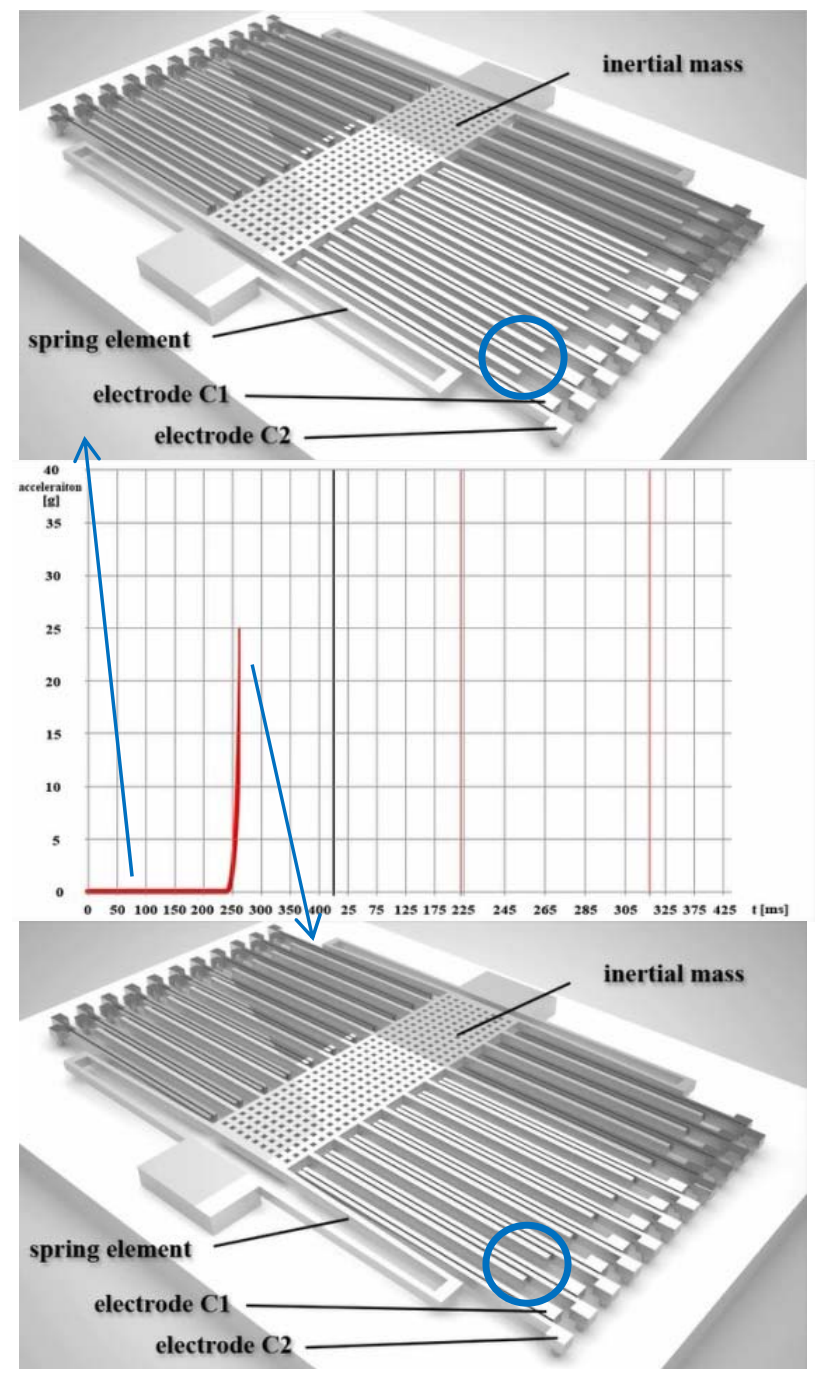

Fig. 4 MEMS accelerometer response of airbag system in idle state closely before an incidence (top) and in time of an impact momentum (bottom) with a barrier 

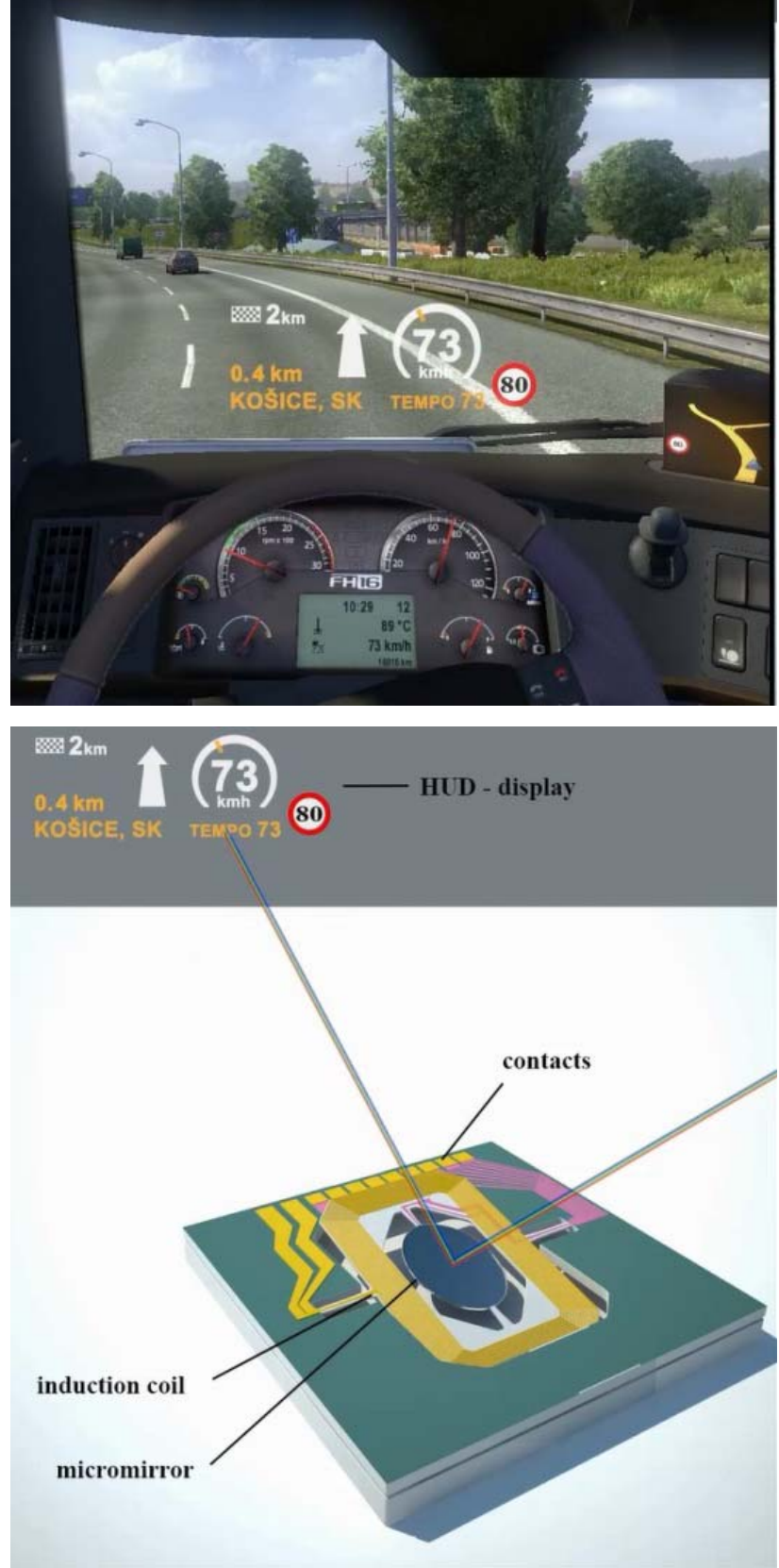

Fig. 5 HUD (head-up display) utilizing MEMS scanner that visualize the navigation, velocity and road signs information on the front window

\section{CONCLUSIONS}

The aim of the work was to utilize the advanced modelling and animation tools for visualization of the structures and working principles of MEMS sensors used in automobile industry and to introduce their problematics in application context for the purpose of education in automobile electronics study subjects.

As the output, a set of animations were created using recent $3 \mathrm{D}$ and $2 \mathrm{D}$ modelling techniques.

Visualization of MEMS accelerometer response in airbag system during an impact with a barrier, the assistant of drive-off system using MEMS inclinometer, head-up display (HUD) utilizing MEMS scanner, measurement of mass-flow (MAF) through intake pipe utilizing MEMS MAF sensor and tire pressure measurement utilizing MEMS pressure gauge are presented.

The animations include photorealistic 3D visualizations of an automobile, detail view to the MEMS sensing microstructure and joined graphical interpretation of sensing response during case situations in which the particular MEMS sensors are utilized in automotive electronics.
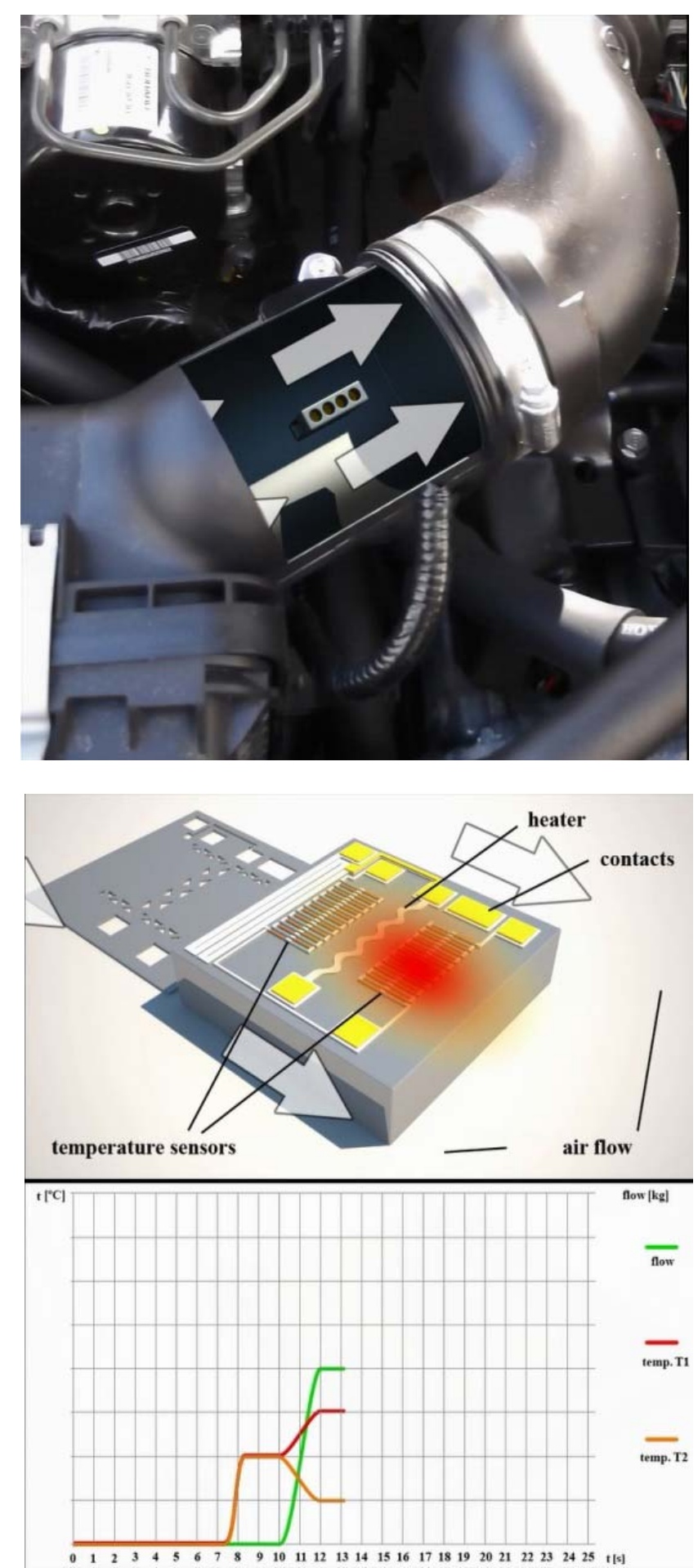

Fig. 6 Measurement of a gaseous working media mass-flow (MAF) in an intake pipe utilizing MEMS MAF sensor during operation with its response to flow of gaseous working media 


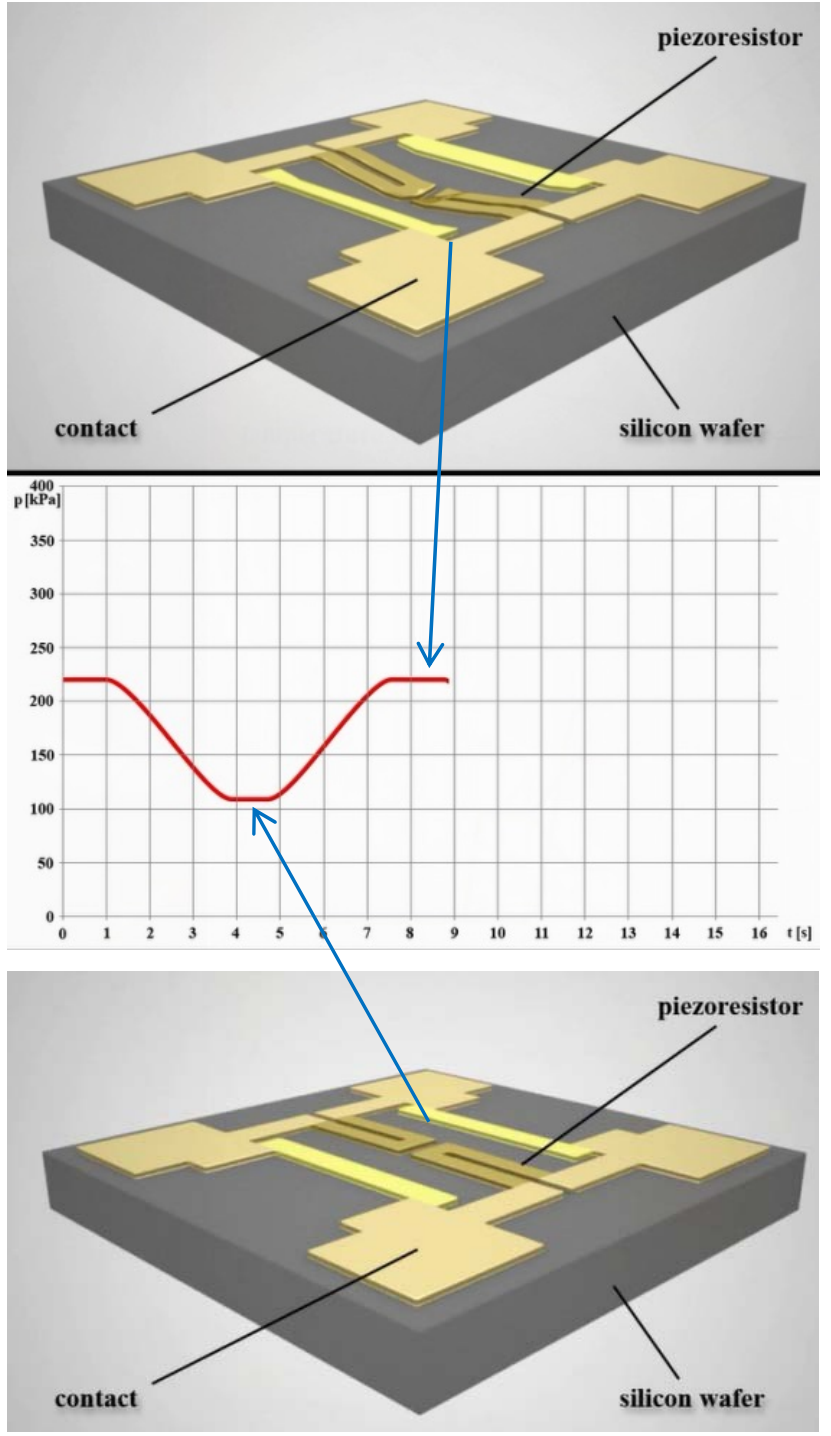

Fig. 7 Visualization of a measurement of tire pressure utilizing MEMS pressure gauge - automobile tire with nominal air pressure (top), underinflated tire (bottom)

\section{ACKNOWLEDGMENTS}

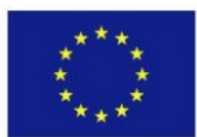

This paper was developed with support of the project "Centre of Excellence of the Integrated Research \& Exploitation the Advanced Materials and Technologies in Európska únia

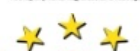

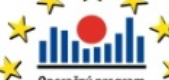
Operanny program 2 头乎为 the Automotive Electronics", ITMS 26220120055, that is co-financed from Structural Funds EU ERDF within Operational programme Research and Development OPVaV-2009/2.1/03-SORO and preferred axis 2 Support of Research and Development.

Special thanks belong to Rudolf Herstek for active participation and highly erudite approach at the work.

\section{REFERENCES}

[1] BRYZEK, J.: Principles of MEMS, Handbook of Measuring System Design, 2005,

$<$ http://eu.wiley.com/legacy/wileychi/hbmsd/pdfs/m m573.pdf $>$

[2] NORIEGA-MANEZ, R. J.: Inertial Navigation, Standford University, 2007,

$<$ http://large.stanford.edu/courses/2007/ph210/norieg a1/>

[3] GANSSLE, P. J.: A Designer's Guide to MEMS Sensors, 2012,

$<$ http://www.digikey.com/es/articles/techzone/2012/j ul/a-designers-guide-to-mems-sensors $>$

[4] HUSÁK, M.: MEMS a mikrosystémové technologie, 2008,

$<$ http://www.odbornecasopisy.cz/index.php?id_docu ment $=38122>$

[5] ISAI, G.: Course Techonolgy of microelectronic devices,

$<$ http://81.161.252.57/ipci/courses/technology/inde 376.htm>

[6] BYU CMR: Introduction to Microelectromechanical Systems (MEMS),

$<$ https://compliantmechanisms.byu.edu/content/intro duction-microelectromechanical-systems-mems $>$

[7] Autodesk: 3DS Max Modelling tutorials, Autodesk Inc., 2017,

$<$ https://knowledge.autodesk.com/support/3dsmax/getting-

started/caas/CloudHelp/cloudhelp/2017/ENU/3DSM ax-Tutorial/files/GUID-C99064E7-7E14-4F989A41-796BFC5613EF-htm.html>

Received June 2, 2017, accepted September 16, 2017

\section{BIOGRAPHIES}

Slavomír Kardoš defended his PhD. thesis in the field of capacitive position sensors. His work is oriented to interconnection techniques, fine thick film technologies, passive components and MEMS technologies.

David Bušek defended his $\mathrm{PhD}$. thesis in the field of electrotechnology, specifically diagnostics of modern electrical joints made by electrically conductive adhesives. His specialization is in soldering technologies and their diagnostics. 